Path. Microbiol. 1965;28:I-XII

\title{
Contents, Vol. 28, 1965
}

Pathologia et Microbiologïa Vol. 28

Pathologia et Microbiologia

Schweiz. Zeitschr. für allg. Pathologie und Bakteriologie

Revue Suisse de Pathologie générale et de Bactériologie

Swiss Journal of General Pathology and Bacteriology

Condiderunt: A. v. ALBERTINI
A. GRUMBACH
H. MOOSER

Offizielles Organ der Schweiz. Mikrobiologischen Gesellschaft Organe officiel de la Société

Suisse de Microbiologie

H. CHIARI, Wien

H. COTTIER, Bern

H. FEY, Bern

J. FIRKET, Liège

C. HALLAUER, Bern

E. LETTERER, Tubingen

J. L. NICOD, Lausanne

COLLABORATORES

A. L. OLITZKI, Jerusalem R. H. REGAMEY, Geneve F. C. ROULET, Basel E.

RUTISHAUSER, Geneve M. et R. SILBERBERG, Saint Louis, Mo.

H. SMETANA, Washington J. TOMCSIK, Basel R. TULASNE, Strasbourg E. UEHLINGER, Zurich M. WELSCH, Liège A.WERTHEMANN, Basel

REDACTORES A. GRUMBACH, Zurich J. R. RÜTTNER, Zurich

Vol. 28

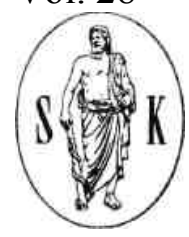

1965

BASEL (Schweiz)

S. KARGER

NEW YORK

Alle Rechte, insbesondere das de $\Gamma$ Übersetzung in fremde Sprachen, vorbehalten.

Ohne ausdrückliche Genehmigung des Verlages ist es auch nicht gestattet, dieses Buch oder

Teile darßus auf photomechanischem Wege (Photokopie, Mikrokopie) zu vervielfãltigen.

(C) 
Copyright 1965 by S. Karger AG., Basel Printed in Switzerland by Buchdruckerei Friedrich Reinhardt AG., Basel Cliches: Aberegg-Steiner \& Cie. AG., Bern, und Steiner \& Cie. AG., Basel Index

Adamiker, D.; Beeitfellnek, G. und Neuhold, R.:

Submikroskopische Verände $\Gamma$ ungen an Staphylokokken nach Tetra-

cyclin-Einwirkung $\quad 460$

Agnihotri, Virendra P.:

The Influence of Carbohydrates on the Growth of Pestalotiopsis versicolor (Speg.) Steyaert 265

Alivisatos, G. P. and Basba, V.:

A New Method for Culturing Amnion Cells 777

Anderson, E. S.: The Work of a National Reference Laboratory

Andries, L.: $\quad$ vide Seeliger, H. P. R.

Arber, W.: $\quad$ Sur le role de la méthylation du DNA dans le contrôle par Thôte de la modification du bactériophage

Bader, G.: $\quad$ vide Schabinski, G.

Barber, Cella; Meitert, Eugenia et Saragea, Alice:

Contributions à $\Gamma$ étude des antigènes endobactériens isolés des ba-

cilles diphtériques; la spécificité des polysaccharides pour Гespèce $\mathrm{C}$.

diphtheriae; antigènes à spécificité de type 274

Basba, V.: $\quad$ vide Alivisatos, G. P.

Bauer, K.: $\quad$ Ein Beitrag zur Clone-Isolierung von Maul- und Klauenseuche-Virus

mittels der Plaquemethode 936

Baumgartner. H.; Kästli, P.; Walser, V. und Meeder, K.:

Versuche über die Bakterizidie der Milch 3

Baumgartner-Morf, R.:

Wirkung verschiedener Alkohole auf das Cytoplasma einer Bacillus-

Spezies 313

Baumgartner-Morf, R.:

Wirkung von Jod und Sublimat auf das Cytoplasma einer Bacillus-

Spezies 469

Bertschinger, H. U. und Scholer, H. J.:

Spontane Cryptococcose beim Meerschweinchen

Bichsel-Werder, E. und Hässig, A.:

Über die Beeinflussung der Serumbakterizidie durch kolloidale Plas-

maersatzlösungen 713

Bienz, K.; Lazãry, S. und Löffler, H.:

Hemmung der Virushämagglutination durch virusinfizierte und nichtinfizierte Mäuselunge;

Beschreibung eines Gewebsinhibitors . . 151

Bloch, H.: $\quad$ vide Kradolfer, F.

Bolle, A.: $\quad$ vide Boy de la Tour, E.

Borowsky, Jerzy and Rutecka, Irena:

Evaluation of Rapid Slide Test for Determination of Staphylococcal

Penicillinase 259

Boy de la Tour, E.; Bolle, A. et Kellenberger, E.: 
Nouvelles observations concernant Taction du laurylsulfate de so dium sur la paroi et la membrane d'E. coli

Boy de la Tour, E.: vide Eiserling, F. A.

Braunwald, Jacqueline; Scherrer, R. et Kirn, A.:

Etude chez le lapin du pouvoir immunisant d'un mutant «froid» de

virus vaccinal 167

Breitfellner, G.: vide Adamiker, D.

Brzin, Bronka : Globular Bodies of Lactobacilli 251

Bürki, F.: $\quad$ Bovine Virus-Diarrhöe. Quantitative Verteilung des Erreger9 und Isolierchancen 158

Bürki, F.: $\quad$ Eigenschaften des Virus der Equinen Arteritis

Çetin, E. T.; Tahsinoglu, M. and Volkan, S.:

Epizootic of Trichophyton Mentagrophytes (Interdigitale) in White

Mice 839

Chatelanat, F. et Simon, G.:

L'atteinte glomérulaire dans les pancréatites aiguës hémorragiques.

Etude aux microscopes optique et électronique $\quad 399$

Chodat, F.: vide Elfiki, $\mathrm{H}$.

Ciucä, M.; Ciplea, Al. Gh. ; Bona, C. et Pozsgi, N.:

La degradation de $\Gamma$ antigène malarique au cours de $\Gamma$ immunogénèse;

enchaînement fonctionnel des facteurs humoraux et cell-ulaires dans

Гimmunité acquise 668

Clerici, E.; Pierpaoli, W. and Romussi, M.:

Experimental Amyloidosis in Immunity 806

Combépine, G. et Turian, G.:

Recherches sur la biosynthèse de la glycine chez Neurospora crassa, type sauvage et mutants 1018

Costin, I. D.: Biochemical Differentiation of Salmonella gallinarum and Salmonella

pullorum cultures 303

Davies, D. A. L.: vide Heidelberger, M.

Diekmann, H.: vide Polli, C.

Diosi, P. and Rosiu, N.:

Cytomegalic Infection in the Submaxillary Glands of an Adult. . . 420

Eiserling, F. A. and Boy de la Tour, E.:

Capsomeres and Other Structures Observed on Some Bacteriophages 175

Elfiki, H. et Chodat, F.:

Exaltation du pouvoir antibiotique de la colimycine par le cobalt . . 114

Engelbrecht, E.: Spécificité et rapidité de la reaction d'hémagglutination passive sur lame 197

Engelbrecht, E.: Les reactions de neutralisation croisée et de neutralisation multiple.

Modifications de la reaction d'hémagglutination passive permettant

une spécificité plus grande de la reaction faite avec des réactifs non

spécifiques 206

Simon, G.: vide Chatelanat, F.

Scholer, H. J.: vide Bertschinger, H. U.

Staehelin, A.: vide Ettlinger, L. 
Starke, G.: $\quad$ Erfahrungen mit den Masernlebendvirusimpfstoffen L-4 und L-16 in der Deutschen Demokratischen Republik 950

Störmer, B.: vide Zur Hausen, H.

Streuli, R. und Ettlinger, L.:

Über einen Kalium-Ammonium-Antagonismus bei Acetobacter per-

oxydans $\quad 1010$

Studer, A. und Lorez, H. P.:

Intravasale Fibrinbildung und ihre Beziehung zur riormalen und ver-

änderten Aorta des Kaninchens 738

Studer, A. und Lorez, H. P.:

Einfluß von Epsilon-Aminocapronsäure auf die nach intravenösen

Thrombininfusionen am Kaninchen entstehenden Veränderungen der

Lungengefäße 425

Tahsinoglu, M.: vide Çetin, E. T.

Tisler, Miha: vide Schauer, Primoz

Tomcsik, J. und Litschel, E.:

Albumin als Faktor in der Senkungsgeschwindigkeit der Blutkörper-

chen 38

Trinh, Phan: Explosion larvaire intra-corporelle des ceufs d'ascaris erratiques

chez un garçon de 4 ans 443

Turian, G.: $\quad$ Différenciation biochimique d'Allomyces: bases nucléiques libres et composition de Tacide ribonucléique $\quad 58$

Turian, G.: vide Combépine, G.

Valladares, Y.: Enzyme-Biologial Synthesis of a cancerigenic Agent ('Cancervirus')

starting from Nucleotides. IV. Metabolic studies with cancerigenic

DNA 761

Valladares, Y.: Enzyme-Biological Synthesis of a Cancerigenic Agent ('Cancervirus')

Starting from Nucleotides, V. Molecular Biology and Genetical

Studies 768

Valladares, Y.: Enzyme-Biological Synthesis of a Carcinogenic Agent ('Cancervirus')

Starting from Nucleotides, III. Biophysico-Chemical Studies with

Cancerigenic DNA and Synthesis of a Cancerigenic DNA Containing

Antimetabolites of the Purine and Pyrimidinc Bases 751

Vertényi, A.: vide Rauss, $\mathrm{K}$.

Viebahn, A.: vide Seeliger, H. P. R.

Vogelsang, Th. M.: Mercury Resistance of Staphylococcus aureus 608

Volkan, S.: vide Çetin, E. T.

Walser, V.: vide Baumgartner, $\mathrm{H}$.

Welsch, M.: Recherches sur des Streptomyces d'Afrique Centrale, V. Production

de pigment vert $\quad 660$

Winkhaus-Schindl, I.:

vide Seeliger, H. P. R.

Engelbrecht, E.: La reaction d'hémagglutination passive, Analyse d'une mosaïque antigénique par les reactions de neutralisation croisée et de neutrali

sation multiple 878

Engelbrecht, E.: Nouvelle reaction pour le diagnostic de la toxoplasmose. La flocula- 
tion sur lame , . 907

Ettlinger, L.; Herrmann, C. und Staehelin, A.:

Morphologie und Teilungsmodus von Pediococcus cerevisiae .... 561

Ettlinger, L.: vide Guggenheim, B.

Ettlinger, L.: vide Jlli, H. U.

Ettlinger, L.: vide Leisinger, Th.

Ettlinger, L.: vide Polli, C.

Ettlinger, L.: vide Streuli, R.

Fey, H.: $\quad$ Fluoreszenz-mikroskopische Identifizierung von abnorm wachsenden Rauschbrand-Clostridien 225

Fey, H.: Vorschlag für eine massenserologische Kontrolle unserer Fremdarbei-

ter auf Typhus und Paratyphus B an der Grenze 626

Friborsky, V.: Induction of Adenoid Cystic Carcinoma of the Submaxillary Gland

in Mice 413

Gard, S.: $\quad$ The Pathogenesis of Bovine Lymphomatosis 683

Gershon, Z.: The Effect of Different Media on Viability, Metabolism and Phago-

cytic Activity of White Cells. Production of a Bactericidal Substance

in the Course of Phagocytosis in vitro 332

Gershon, Z.: The Effect of Phagocytosis on the Succinic Dehydrogenase Activity

of Mouse Polymorphonuclears Determined Macroscopically and

Histochemically 501

Gershon, Z.: vide Olitzki, A. L.

Gindrat, J. J.: vide Hazeghi, K.

Gouda, S.: $\quad$ Oxydo-réductibilité du pigment de Pseudomonas fluorescens in vivo

et in vitro 107

Grün, L.: vide Kikuth, W.

Guggenheim, B.; Ettlinger, L. und Mühlemann, H. R.:

Die Wirkung von flüchtigen Säuren im Speichel auf den aeroben

Metabolismus von Mundbakterien 77

HÄssiG, A.: vide Bichsel-Werder, E.

Hannouz, M.: Antigénicité et pureté d'une preparation de lysozyme

Hardmeier, Th. : Zur Differentialdiagnose der Pigmentierungen des Verdauungstraktes 437

Hauduroy, P.: De Thétérogénéité des populations bactériennes $\quad 602$

Hauduroy, P. et Rosset, W.:

B.C.G. et infections provoquées chez des animaux de laboratoire . 992

Hazeghi, K. et Gindrat, J. J.:

Sérobactériolyse non spécifique et antibiorésistance acquise chez E.

coli 122

Heidelberger, M.; Rao, C. V. N. and Davies, D. A. L.:

The Polysaccharide of Shigella dysenteriae and its Cross-Reactions

with Antisera to the Pneumococcal Types II and VI 691

Herrmann, Chr. : Über die zugleich quantitative DifFerenzierung von erhitztem Eiweiß mittels der indirekten Hämagglutination $\quad 886$

Herrmann, C.: vide Ettlinger, L.

Hudemann, H.: Häufigkeit und diagnostische Bedeutung von Typhus-Vi-Phagen beim

Menschen 181 
Hummel, K.: Über die Wirkung von Cholera-Neuraminidase im Vergleich zu anderen Neuraminsäure abspaltenden Fermenten pflanzlicher und tierischer Herkunft auf einige physiokochemische Eigenschaften menschlicher Erythrozyten 891

Hütter, R.: $\quad$ Die Gattung der Actinomycetales 567

Idriss, M.: $\quad$ Study on the Combined Effect of Neomycin and Ampicillin on Salmonellae $\quad 130$

IllÉs, J.: $\quad$ Der Koagulase-Aktivatorgehalt des Meerschweinchenplasmas und die Hitzeresistenz des Aktivators 834

Jlli, H. U.; Müller, J. und Ettlinger, L.:

Der Einfluß der C-Quelle auf den Wuchsstoff bedarf von Essigsäurebakterien 1005

Kästli, P.: $\quad$ vide Baumgartner, $\mathrm{H}$.

Kauffmann, F.: Die Diagnose von Arizona-Kulturen nach dem originalen KauffmannWhite-Schema 575

Kayser, F. H.: Mutatorfaktor bei Staphylococcus pyogenes aureus? 985

Kellenberger, E.: vide Boy de la Tour, E.

Kellenberger, E.: Die Elektronenmikroskopie in Molekularbiologie und Mikrobiologie. 540

Keller, R.: Zur Bedeutung von Lysolecithin für die Immuncytolyse von Mastzellen 185

Kéty, I.: $\quad$ vide Rauss, K.

Kikuth, W. und Grün, L.:

Zur Epidemiologie und Biologie von Aerobacter aerogenes .... 632

Kirn, A.: $\quad$ vide Braunwald, Jacqueline

Kis, Z.: $\quad$ vide Polli, C.

Klein, P. A. and Lindenmann, J.:

Further Studies on the Soluble c-Antigen of the Mouse 698

Koenig, M.: Einfache Labormethode zur Prüfung des Blockierungseffektes bei

Bacteriostatica und Fungistatica 997

Kókai, K.: vide KovÁcs, E.

KovÁcs, E. und Márton, K. P.:

Die Wirkung der Askorbinsäure auf die Redoxkapazität und die Ent-

wicklung von Streptomyces griseus-Kulturen 296

KovÁcs, E. und Kókai, K.:

Eine Methode zur schnellen elektrometrischen Bestimmung der Anti-

biotikumempfindlichkeit der Bakterien $\quad 454$

KovÁcs, E.: vide Matkovics, B.

Kôvári, I.: vide Matkovics, B.

Kradolfer, F. and Bloch, H.:

Intermittent Combination Therapy with INH and Thiambutosine

(CIBA-1906) in Tuberculous Mice 717

Khbavcic, Ales: vide Schauer, Primoz

Laue, F.: $\quad$ Ergebnisse der serologischen Ornithose-Diagnostik 214

Lazáry, S.: $\quad$ vide Bienz, K.

Leisinger, Th. und Ettlinger, L.:

Über die Kohlenstoffernährung von Essigsäurebakterien 84

Leopold, P. G.: vide Wohlgemuth, B. 
Likar, Miha: vide Schauer, Primoz

Likar, Miha: vide Schauer, Primoz

Lindenmann, J.: vide Klein, P. A.

Litschel, E.: vide Tomcsik, J.

Löffler, H.: $\quad$ vide Bienz, K.

Lorez, H. P.: vide Studer, A.

Lorez, H. P.: vide Studer, A.

Lwoff, A.: Role des facteurs non spécifiques au cours de Tinfection virale primaire 147

Mai, K. und Roemer, G. B.:

Indirekte Hämagglutination durch Antikörper gegen Streptokokken-

Gruppensubstanz D 901

Marmur, J.: New Approaches to Bacterial Taxonomy

Márton, K. P.: vide KovÁcs, E.

Matkovics, B.; KovÁcs, E. and Kõvári, I.:

The Effect of Catalase on the Redox-changes of Streptomyces griseus

134-Culture, Preliminary Communication 830

Mechsner, Kl.: vide Wuhrmann, K.

Meeder, K.: $\quad$ vide Baumgartner, $\mathrm{H}$.

Megnet, R.: Alkoholdehydrogenasemutanten von Schizosaccharomyces pombe . 50

Meitert, Eugenia : vide Barber, Cella

Mühlemann, H. R.: vide Guggenheim, B.

Müller, J.: $\quad$ vide Jlli, H. U.

Neipp, L.: $\quad$ vide Sackmann, W.

Neter, E.: $\quad$ Indirect Bacterial Hemagglutination, and its Application to the

Study of Bacterial Antigens and Serologic Diagnosis $\quad 859$

Neuhold, R.: vide Adamiker, D.

Olitzki, A. L. and Gershon, Z.:

Splenocytin, a Protective and Curative Product of Murine Spleen Cells 704

Oít, A.: $\quad$ vide Schär, M.

Penso, G.: $\quad$ Mycobacterium minetti (Penso et al., 1951) 581

Pierpaoli, W.: vide Clerici, E.

Pollak, Alfred: vide Schauer, Primoz

Polli, C; Diekmann, H.; Kis, Z. und Ettlinger, L.:

Über das Vorkommen von Ketonreduktasen bei Mikroorganismen 93 Primavesi, C. A.:

Virologische Untersuchungen an Abwässern und Flußwässern . . 956 Rauss, K.; Kétyi, I. and Vertényi, A.:

New Observations on the Production of Precipitated Typhoid Vaccine 357

Rao, C. V. N.: vide Heidelberger, M.

Reekers, A. and Wikén, T. O.:

On the Production of a-Ketoglutaric Acid by Non-Proliferating Cells

of Pseudomonas reptilivora Caldwell et Ryerson from Cultures of

Different Age 648

Richtarik, A. A. and Seager, L. D.:

Studies on the Combined Action of Stilbamidine and Penicillin in Ex

perimentally Induced Bacterial Endocarditis 847

Roemer, G. B.: vide Mai, K. 
Romussi, M.: vide Clerici, E.

Rosiu, N.: vide Diosi, P.

Rutecka, Irena: vide Borowski, Jerzy

Sackmann, W. und Neipp, L.:

Dosishäufigkeit und Versuchsdauer bei der experimentellen Chemo-

therapie bakterieller Infektionen der Maus 26

Saez, H.: $\quad$ Les geotrichum à $\Gamma$ origine de mycoses profondes? 287

Saragea, Alice : vide Barber, Cella

Sarbhoy, A. K.: Nutritional Studies on some Members of the Mucorales. V. 1. Utiliza

tion of Different Nitrogen Compounds $\quad 816$

Seager, L. D.: vide Richtarik, A. G.

Seeliger, H. P. R.; Winkhaus-Schindl, I.; Andries, L. und Viebahn, A.:

Die Isolierang von Listeria monocytogenes aus Stuhl-, Klär-

schlamm- und Erdproben. (Mit einem Hinweis auf die Epidemiologie

der Listeriose) 590

Schabinski, G. und Bader, G.:

Möglichkeiten, Bewertung und Grenzen des Nachweises von Pilzen

im Gewebe. Experimenteller Vergleich zwischen kultureller und histo-

logischer Pilzdiagnostik 487

Schäfer, W.: Wechselwirkungen zwischen tierpathogenen Virusarten und ihren

Wirtszellen 917



Die Tuberkulosebekämpfung in epidemiologischer Sicht 639

Schauer, Primoz and Likar, Miha

Studies of some Substances with Antiviral Activity; I. The Inhibitory

Effect of Guanidine on the Growth of Herpes Simplex Virus in Human

Embryonic Kidney Cell Cultures 371

Schauer, Primoz; Likar, Miha; Tisler, Miha; Krbavcic, Ales and Pollak, Alfred:

Studies of some Substances with Antiviral Activity. II. 2,4-Dioxo-5-

Thiazolidine Acetic Acid Derivatives (DFT) as an Inhibitor of

Growth of Herpes Virus and Poliovirus Type I in Cell Cultures of

Human Embryonic Kidneys 382

Scherrer, R.: vide Braunwald, Jacqueline

Schmidt, J.: Untersuchungen über den fluoreszenzserologischen Nachweis von

Pasteurella pseudotuberculosis-Antikörpern 21

Schmidt, J.: Vorkommen von Staphylococcus aureus-Mehrfachinfektionen bei geschlossenen und offenen Eiterprozessen $\quad 974$

Wohlgemuth, B. und Leopold, P. G.:

Morbus Bang und Splenektomie 729

Wood, W. B.: Mutations in E. coli Affecting the Host-Controlled Modification of

Bacteriophage $\lambda$ 73

Wuhrmann, K. und Mechsner, K1. :

Über den Einfluß von Sauerstoffspannung und Wasserstoffionen-

konzentration des Milieus auf die mikrobielle Denitrifikation ...

99

Zangger, J.: Eine neue Methode der Empfindlichkeitsprüfung von Sulfonamiden:

«Zwei-Röhrchen-Indikator-Test» 797 
Zschiesche, W.: Ölinduzierte Amyloidose bei Cancerogenversuchen mit Cholesterinderivaten 393

Zur Hausen, H. und Störmer, B.:

Cytogenetische Untersuchungen an L-Zellen nach Vaccinia-Virus-

Infektion 962

Verhandlungsberichte - Proceedings - Congrès

Société Suisse de Microbiologie, 23e Assemblée annuelle, du 6 au 7

juin 1964, à Geneve - Schweizerische Mikrobiologische Gesellschaft,

23. Jahresversammlung, 6.-7. Juni 1964 in Genf

1

Schweizerische Mikrobiologische Gesellschaft, 24. Jahresversamm

lung, 11.-13. Juni 1965 in Luzern - Société Suisse de Microbiologie,

24e Assemblée annuelle, du 11 au 13 juin 1965 à Lucerne 857

Mitgliederliste der Schweizerischen Mikrobiologischen Gesellschaft -

Membres de la Société Suisse de Microbiologie 236,1035

Personalia: Prof. Dr. A. Grumbach - Festschrift zum 70. Geburtstag gewidmet, 25. Juni1965

Prof. Dr. A. Grumbach - Anniversary Issue, dedicated to his 70th

birthday, June 25, 1965

Buchbesprechungen - Book Reviews - Livres Nouveaux 243, 388, 512, 520, 728, 853, 1042

Varia 520

Nekrologia: $\quad$ Prof. Dr. Georg Sobernheim (1865-1962) zum Gedächtnis 235

Dr. Jean Clerc 1031

Prof. Dr. W. H. Schopfer (1900-1962) 1032

Index rerum 1044

Index autorum 1065 